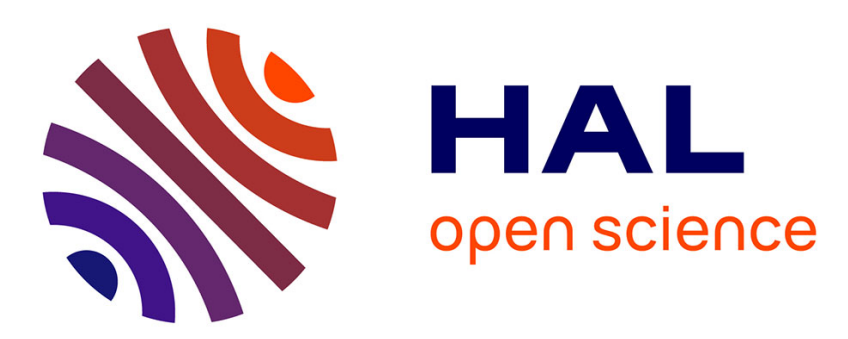

\title{
Nitrure d'aluminium de différentes morphologies obtenu à basse température par PACVD
}

Jean Durand, Nathalie Azéma, Bemard Cros, Louis Cot

\section{To cite this version:}

Jean Durand, Nathalie Azéma, Bemard Cros, Louis Cot. Nitrure d'aluminium de différentes morphologies obtenu à basse température par PACVD. Journal de Physique III, 1993, 3 (4), pp.713-727. 10.1051/jp3:1993158 . jpa-00248952

\section{HAL Id: jpa-00248952 https://hal.science/jpa-00248952}

Submitted on 1 Jan 1993

HAL is a multi-disciplinary open access archive for the deposit and dissemination of scientific research documents, whether they are published or not. The documents may come from teaching and research institutions in France or abroad, or from public or private research centers.
L'archive ouverte pluridisciplinaire HAL, est destinée au dépôt et à la diffusion de documents scientifiques de niveau recherche, publiés ou non, émanant des établissements d'enseignement et de recherche français ou étrangers, des laboratoires publics ou privés. 
Classification

Physics Abstracts

$68.22-68.48-68.55-81.15 \mathrm{H}$

\title{
Nitrure d'aluminium de différentes morphologies obtenu à basse température par PACVD
}

\author{
Jean Durand, Nathalie Azéma, Bernard Cros et Louis Cot \\ Laboratoire de Physicochimie des Matériaux, URA CNRS 1312 ENSCM, 8 rue de l'Ecole \\ Normale, 34053 Montpellier Cedex 1, France
}

(Reçu le 25 mai 1992, révisé le 3 novembre 1992, accepté le 10 novembre 1992)

\begin{abstract}
Résumé. - Le dépôt chimique en phase vapeur d'organo-métalliques assisté d'un plasma réactif conduit à des revêtements cristallins de nitrure d'aluminium ayant une orientation préférentielle $(10 \overline{1} 0)$ marquée. La dimension des cristallites dépend de la fréquence d'excitation plasma et est indépendante de la nature du substrat. Les revêtements, caractérisés par diffraction des rayons $\mathrm{X}$, spectroscopies infrarouge et des électrons Auger, montrent une composition AlN. L'interphase AIN/Si a fait l'objet d'une étude particulière par microscopie électronique à transmission.

Abstract. - The metal-organic chemical vapor deposition process assisted by a reactive plasma leads to crystalline AlN coatings with a preferential orientation (10 $\overline{1} 0$ ). The crystallite size strongly depends on the plasma frequency and is independent of substrate materials. The coatings have been characterized by X-ray diffraction, infrared and Auger spectroscopies. The AlN/Si interphase has been studied by transmission electron microscopy.
\end{abstract}

\section{Introduction.}

Le nitrure d'aluminium est une céramique dont les propriétés physiques, thermiques, mécaniques et chimiques forment une combinaison particulièrement attrayante. De ce fait, AlN devient un candidat potentiel pour des utilisations très variées : microélectronique, optique, ondes acoustiques de surface, piézoélectricité, électronique de puissance...

Depuis quelques années la réalisation de ce matériau sous forme de couche mince fait l'objet d'une attention particulière. Les revêtements d'AlN peuvent être obtenus sous forme épitaxiée, polycristalline ou amorphe. Selon les méthodes et les conditions de préparation la structure des matériaux obtenus peut être très différente. Le dépôt chimique en phase vapeur, l'épitaxie réactive par jet moléculaire ou la pulvérisation cathodique réactive $R F$ effectués à des températures comprises entre 800 et $1200^{\circ} \mathrm{C}$ conduisent à des films cristallins pour lesquels une orientation préférentielle (0001) est très marquée. Par contre, en utilisant la méthode de dépôt chimique en phase vapeur assisté d'un plasma réactif (PACVD), Hasegawa [1] a obtenu des films minces amorphes à $300^{\circ} \mathrm{C}$.

Dans ce travail, la méthode PACVD nous a permis de préparer, à $300^{\circ} \mathrm{C}$, des films minces de nitrure d'aluminium ayant une orientation préférentielle $(10 \overline{10})$ très marquée sur une large 
gamme de substrats. Nous présentons ici les résultats concernant l'influence de la fréquence d'excitation plasma sur la cristallinité des dépôts obtenus. Ces films minces sont caractérisés par diffraction des rayons $X$, spectroscopie d'absorption infra-rouge et spectroscopie des électrons Auger. Une étude complémentaire nous a permis de mettre en évidence la présence d'une interphase complexe entre la couche de nitrure d'aluminium et un substrat de silicium (100).

\section{Généralités.}

Le nitrure d'aluminium est le seul composé existant dans le diagramme aluminium-azote [2]. C'est un nitrure covalent, dont le degré d'ionicité de 0,449 est toutefois appréciable. Son point de fusion est supérieur à $2200^{\circ} \mathrm{C}$ sous pression d'azote, sa densité théorique de 3,26 et son indice de réfraction de 2,17 .

Il cristallise dans le système hexagonal, groupe d'espace $P 6_{3} \mathrm{mc}$, avec les paramètres :

$$
a=3,111 \AA \quad c=4,978 \AA .
$$

Les distances interatomiques et les angles de liaisons sont rassemblées dans le tableau suivant. Ce composé est stable chimiquement et non toxique.

Distances et angles interatomiques dans AlN

$$
\begin{aligned}
& A \mathrm{l}-\mathrm{N}^{\prime}=1,917 \AA \\
& \mathrm{Al}-\mathrm{N}=1,885 \AA \\
& \mathrm{N}^{\prime}-\mathrm{Al}-\mathrm{N}=107,7^{\circ} \\
& \mathrm{N}-\mathrm{Al}-\mathrm{N}=110,5^{\circ}
\end{aligned}
$$

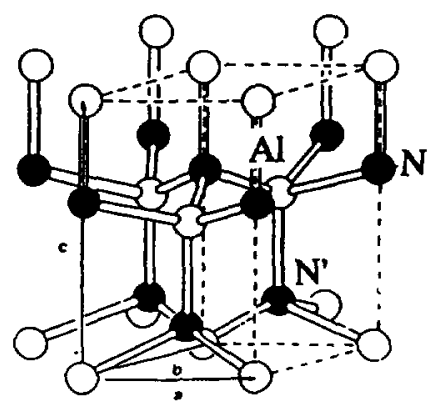

Ses propriétés physiques:

Résistivité volumique

Tension de claquage

Constante diélectrique à $1 \mathrm{MHz}$

Largeur de bande interdite [3, 4]

Absorption optique centrée [3] sur

Conductivité thermique

Coefficient de dilatation thermique

Coefficient électro-optique [5]

Vitesse des ondes de surface [6]

Coefficient de couplage électromécanique

Coefficient de susceptibilité non linéaire [7]

Dureté vickers

Module d'élasticité

Résistance à la flexion $[8,9]$
$>10^{13} \Omega . \mathrm{cm}$

$10 \mathrm{kV} / \mathrm{cm}$

8,9

$5,9-6,2 \mathrm{eV}$

$2,8 \mathrm{eV}$

$320 \mathrm{~W} / \mathrm{m} . \mathrm{K}$ à $25^{\circ} \mathrm{C}$

$3,5 \times 10^{-6} / \mathrm{K}$ à $200^{\circ} \mathrm{C}$

$r_{13}=0,67 \mathrm{pm} / \mathrm{V}$

$r_{33}=-0,59 \mathrm{pm} / \mathrm{V}$

$V_{\text {trans }}=6-6,2 \mathrm{~km} / \mathrm{s}$

$V_{\text {long }}=11-12 \mathrm{~km} / \mathrm{s}$

$K^{2}>1 \%$

$d_{31} / d_{33}<0,04$

$1200 \mathrm{~kg} / \mathrm{mm}^{2}$

$300 \mathrm{kN} / \mathrm{mm}^{2}$

$5000 \mathrm{~kg} / \mathrm{cm}^{2}$

en font un candidat particulièrement intéressant pour des applications dans les domaines de la protection thermomécanique, l'électronique de puissance, les systèmes acousto-optiques et électro-optiques. 


\section{Partie expérimentale.}

3.1 CONDITIONS DE DÉPÔT. - Les couches minces de nitrure d'aluminium ont été préparées par dépôt chimique en phase vapeur assisté d'un plasma réactif dans un réacteur à murs chauds, de type capacitif, à électrodes internes décrit précédemment [10]. Les conditions de synthèse ont été tenues constantes : pression totale $=7 \mathrm{~Pa}$, densité de puissance plasma appliquée = $0,26 \mathrm{~W} / \mathrm{cm}^{2}$, débits des gaz réactifs (triméthylealuminium, ammoniac et azote avec $\left.p\left(\mathrm{NH}^{3}\right) / p(\mathrm{TMA}+\mathrm{N} 2)>3\right)$ et température $=330^{\circ} \mathrm{C}$.

Différentes valeurs de la fréquence d'excitation plasma ont été utilisées en vue d'étudier l'influence de ce paramètre sur les caractéristiques des dépôts. Les différentes fréquences sont $35,110,440 \mathrm{kHz}$ et $13,56 \mathrm{MHz}$.

\subsection{CARACTÉRISTIQUES DES FILMS.}

Microscopie électronique à balayage. - Les photographies de la figure 1 montrent des couches d'AlN de $2 \mu \mathrm{m}$ d'épaisseur sur des substrats de silicium monocristallin et de graphite. $L$ 'aspect appraît très uniforme et très dense avec une excellente adhérence au substrat et un très bon pouvoir de recouvrement. La surface présente peu de défaut.
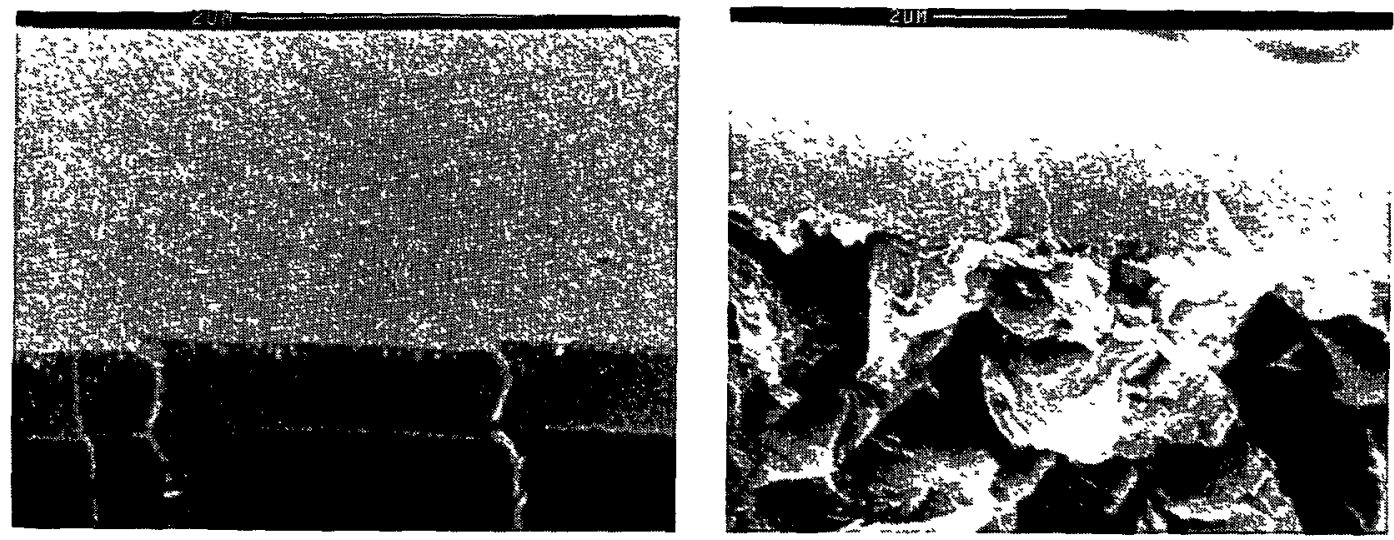

Fig. 1. - Couche mince d'AlN déposé sur silicium et graphite.

[AIN coating on silicon and graphite substrates.]

Spectroscopie d'absorption infrarouge (FTIR). - Tous les spectres montrent un pic intense à $670 \mathrm{~cm}^{-1}$ caractéristique des vibrations d'élongation de la liaison Al-N. Deux autres bandes d'absorption ont été identifiées et correspondent aux vibrations $\mathrm{N}-\mathrm{H}\left(3205 \mathrm{~cm}^{-1}\right)$ et $\mathrm{Al}-\mathrm{H}$ $\left(2108 \mathrm{~cm}^{-1}\right)$. Le matériau obtenu est donc hydrogéné et l'hydrogène peut provenir soit de l'ammoniac, soit du triméthylealuminium. La quantité d'hydrogène a été estimée par analyse nucléaire : sa concentration atomique est de $17 \%$.

Spectroscopie d'électrons Auger. - Après un décapage de la surface par un bombardement avec des ions $\mathrm{Ar}^{+}$, les spectres sont enregistés en conservant ce faisceau ionique. Ainsi, les couches minces étudiées montrent peu d'impuretés, environ $2 \%$ de carbone et nettement moins de $1 \%$ d'oxygène. Un spectre caractéristique est représenté sur la figure 2 . la détermination du rapport atomique $\mathrm{Al} / \mathrm{N}$ montre qu'il est très voisin de 1.

Ellipsométrie. - L'indice de réfraction des films déposés sur du silicium a été mesuré sous une incidence de $30^{\circ}$. L'indice varie entre 2,0 et 2,2 pour une longueur d'onde de $633 \mathrm{~nm}$. 


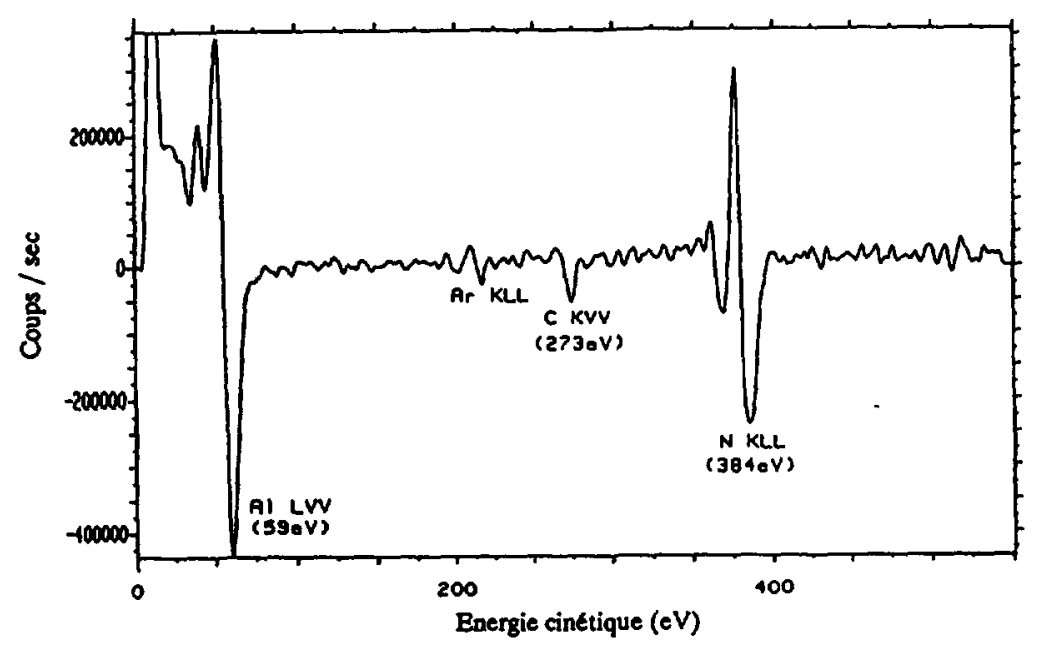

Fig. 2. - Spectre AES basse énergie d'AIN.

[AES low energy spectrum of AIN.]

La mesure de la densité $(d=2,6)$ par la méthode de flottation complète cette caractérisation générale de nos films minces.

\section{Influence de la fréquence d'excitation.}

4.1 CINÉTIQUE DE DÉPÔT. - La figure 3 présente la variation d'épaisseur des films obtenus en fonction du temps de dépôt pour trois fréquences d'excitation utilisées : $35,440 \mathrm{kHz}$ et 13,56 MHz. On constate que la vitesse de croissance diminue lorsque la fréquence augmente. Ces différences sont dues principalement à l'effet du bombardement ionique par les ions

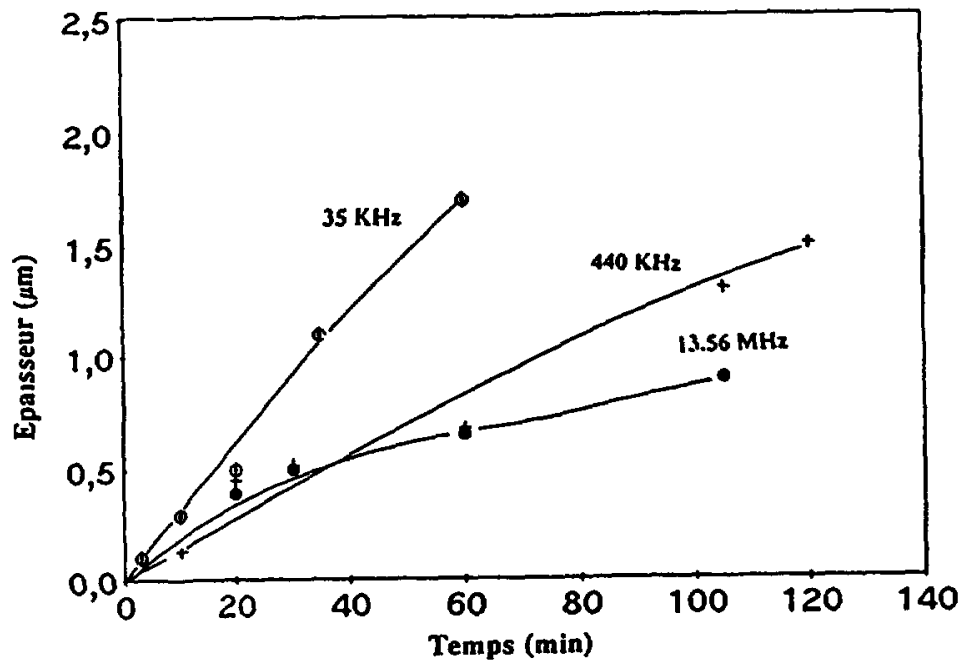

Fig. 3. - Influence de la fréquence d'excitation sur la vitesse de dépôt.

[Growth rate for different excitation frequencies.] 
positifs créés dans la décharge électroluminescente. Leur flux augmente et leur énergie diminue lorsque la fréquence augmente. Ceci cause des changements dans la cinétique de croissance, la structure, la composition et les contraintes résiduelles dans le film. Un bombardement par des ions très énergétiques (basse fréquence) crée un nombre important de sites de croissance et ainsi la vitesse de dépôt augmente. Nous vérifierons plus loin que la composition des films n'est que peu modifiée par ce changement de vitesse de croissance.

\subsection{DIFFRACTION DES RAYONS $X$.}

Influence de la fréquence sur la taille des grains. - Nous utilisons un compteur courbe INEL pour la caractérisation par rayons $X$ des couches préparées. La figure 4 montre de façon significative les différences dans la cristallinité des films produits à basse et haute fréquence d'excitation rf. Tous les autres paramètres de dépôt sont identiques. Pour les fréquences 35 , 110 et $440 \mathrm{kHz}$, les diffractogrammes de rayons $X$ indiquent une orientation préférentielle des cristallites selon la direction (10 $\overline{1} 0$ ). Par contre, lorsque la fréquence d'excitation est de 13.56 $\mathrm{MHz}$ aucune raie de diffraction n'est observée, ce qui est caractéristique d'un matériau amorphe ou d'un matériau dont les cristallites ont des dimensions très faibles (inférieures à quelques nanomètres). Cette observation est en parfait accord avec les résultats d'Hasegawa [1].

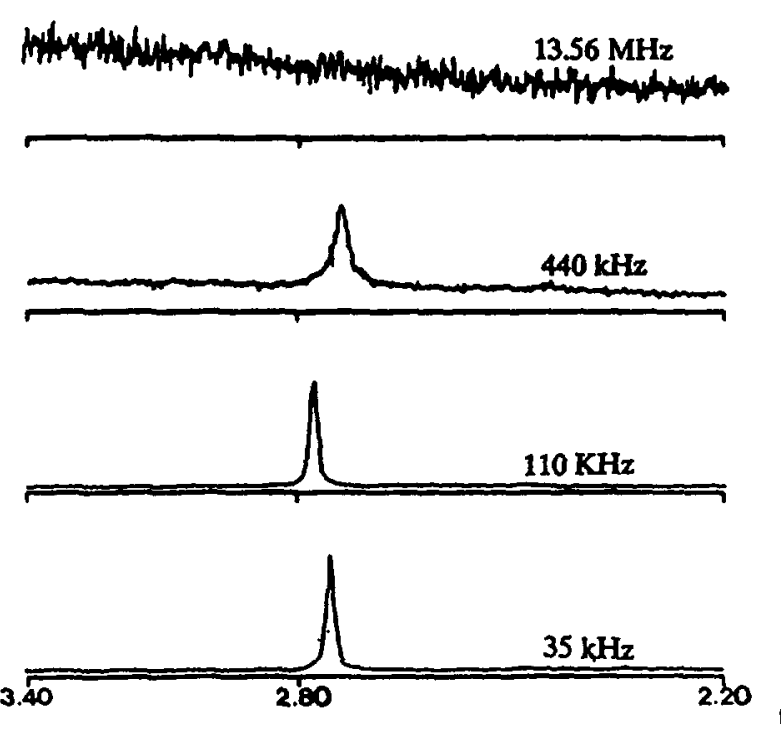

Fig. 4. - Influence de la fréquence d'excitation sur la cristallinité des dépôts d'AlN.

[Influence of the excitation frequency on the AlN thin film crystallinity.]

Une étude sur la dimension moyenne des cristallites a été réalisée à partir de la mesure de la largeur à mi-hauteur du pic de diffraction. Pour effectuer cette étude nous avons utilisé la relation de Scherrer [11] communément admise :

$$
L=(0,9 \lambda) /\left(B \cos \theta_{B}\right)
$$

dans laquelle $\lambda$ est la longueur d'onde du rayonnement $\mathrm{X}$ utilisé $\left(\lambda\left(\mathrm{Cu} \mathrm{K} \alpha_{1}\right)==1,5418 \AA\right)$, $\theta_{\mathrm{B}}$ l'angle de Bragg et $B$ l'élargissement de la raie considérée (dû à la faible taille des particules). Cet élargissement est déduit de la relation de Warren [11] :

$$
B^{2}=B_{\mathrm{M}}^{2}-B_{\mathrm{S}}^{2}
$$


où $B_{M}$ et $B_{\mathrm{S}}$ représentent les largeurs à mi-hauteur des raies considérées, respectivement pour le film étudié et l'échantillon de référence (raie (104) de l'alumine $\alpha$ de granulométrie 5,6 $\mathrm{\mu m}$ ).

Les résultats consignés dans le tableau $I$ montrent que les cristallites ont des dimensions inférieures à $600 \AA$ et que ces dimensions sont peu influencées par l'épaisseur de la couche (en particulier pour les films obtenus à $35 \mathrm{kHz}$ ). Pour une fréquence de $440 \mathrm{kHz}$ la dimension moyenne est d'environ $260 \AA$. Cette diminution est en désaccord avec les résultats de Matsuda [12] qui observe une augmentation de la taille des cristallites de silicium avec l'énergie du bombardement ionique correspondant à une diminution de la fréquence d'excitation plasma.

Tableau I. - Evolution de la taille $(L)$ des cristallites avec la fréquence.

[Mean values of the crystallite size $(L)$ for different frequencies.]

\begin{tabular}{|l|c|c|c|c|}
\hline \multicolumn{1}{|c|}{$\begin{array}{c}\text { Echantillons } \\
(\text { Epaisseur en } \mu \mathrm{m})\end{array}$} & $\theta_{\mathrm{B}}\left(^{\circ}\right)$ & $B_{\mathrm{S}}$ ou $B_{\mathrm{M}}\left(^{\circ}\right)$ & $B(\AA)$ & $\begin{array}{c}L(\AA) \\
(+/-50 \AA)\end{array}$ \\
\hline Alumine $\alpha$ & 17,45 & $B_{\mathrm{S}}=0,14$ & $\begin{array}{c}\text { granulométrie } \\
=5,6 \mu \mathrm{m}\end{array}$ \\
\hline $\begin{array}{l}\text { AlN-35 kHz } \\
(0,5)\end{array}$ & 16,24 & $B_{\mathrm{M}}=0,21$ & $B=0,16$ & 530 \\
$\begin{array}{l}\text { AIN-35 kHz } \\
(1,2)\end{array}$ & 16,32 & $B_{\mathrm{M}}=0,20$ & $B=0,14$ & 580 \\
AlN-35 kHz & 16,40 & $B_{\mathrm{M}}=0,22$ & $B=0,17$ & 490 \\
$\begin{array}{l}\text { AlN-110 kHz } \\
\text { AlN-440 kHz }\end{array}$ & 16,13 & $B_{\mathrm{M}}=0,21$ & $B=0,16$ & 500 \\
\hline AlN (pulvérisation cathodique [34]) & \multicolumn{2}{|c|}{ orientation cristalline $(0002)$} & 260 \\
\hline
\end{tabular}

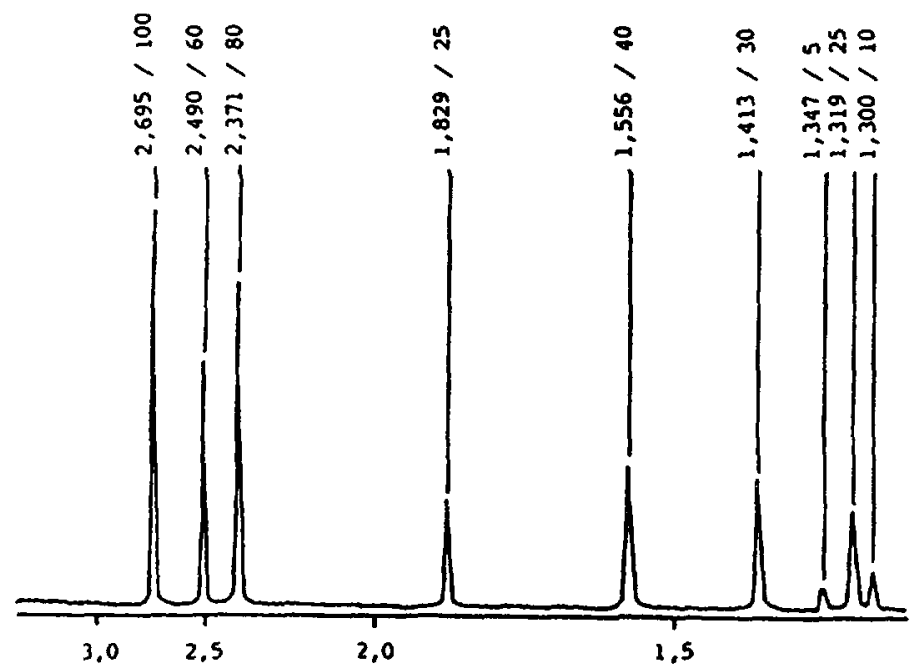

Fig. 5. - Diffractogramme de poudre d'AlN.

[X-ray diffraction pattern of AlN powder.] 
Effet du substrat. - L'utilisation de substrats de nature, de cristallinité et de rugosité différentes nous a permis de confirmer que les films sont obtenus avec l'orientation préférentielle (10īo) très marquée. La figure 6 montre les diffractogrammes de couches déposées sur des substrats de silicium monocristallin d'orientation (100) et (111), de carbure de silicium fritté (dans ce cas seule la raie pointée appartient à $\mathrm{AlN}$, les autres sont celles de $\mathrm{SiC})$ et de verre. Dans tous les cas on observe uniquement la raie de diffraction $(10 \overline{1} 0)$.
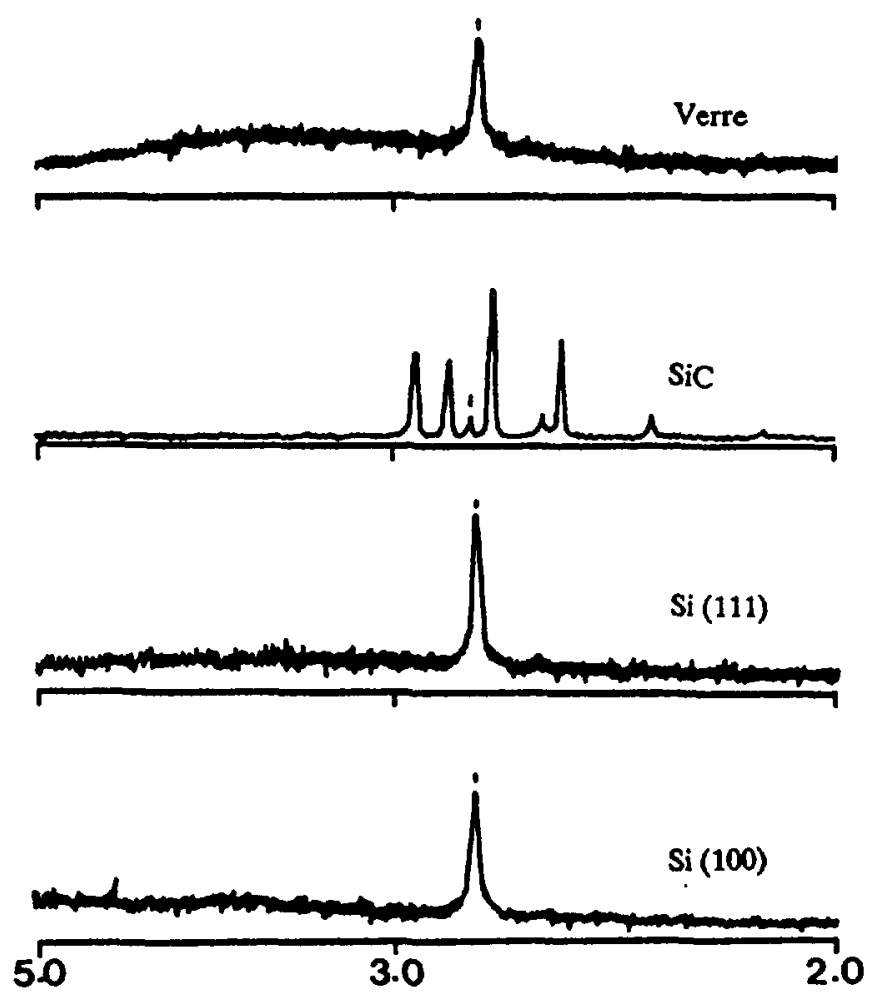

Fig. 6. - Influence de la nature du substrat sur l'orientation cristalline.

[Influence of the substrate on AlN crystalline orientation.]

Le tableau II montre également que la dimension des cristallites est homogène et égale à environ $260 \AA$ pour des couches obtenues avec une fréquence d'excitation de $440 \mathrm{kHz}$.

Il apparaît donc que, dans des conditions de dépôt bien déterminées, la nature et la rugosité du substrat n'a aucune influence sur la cristallinité de la couche.

4.3 SPECTROSCOPIE D'ABSORPTION INFRA-ROUGE. - L'observation de la bande d'absorption infra-rouge Al-N à $670 \mathrm{~cm}^{-1}$ confirme les résultats obtenus en diffraction des rayons $X$. Lorsque la fréquence d'excitation augmente, on observe un élargissement de cette bande dû à la diminution de la taille des cristallites (Fig. 7).

De même, l'analyse des bandes Al-H et $\mathrm{N}-\mathrm{H}$ montre que l'intensité de ces bandes d'absorption subit une évolution lorsque la fréquence varie. La figure 8 donne l'évolution de l'intensité de ces deux bandes d'absorption ramenées à une même épaisseur. Si l'intensité de la bande $\mathrm{N}-\mathrm{H}$ varie peu, celle de la bande Al-H augmente nettement pour une fréquence de $13,56 \mathrm{MHz}$ ce qui est révélateur d'une fixation préférentielle de l'hydrogène sur l'aluminium 
Tableau II. - Dimension des cristallites d'AlN synthétisé à $440 \mathrm{kHz}$ sur différents substrats. [Mean values of crystallite size $(L)$ of AIN for two experiments.]

\begin{tabular}{|l|c|c|c|c|}
\hline \multicolumn{1}{|c|}{ Substrats } & $\theta_{\mathrm{B}}\left(^{\circ}\right)$ & $B_{\mathrm{M}}\left(^{\circ}\right)$ & $B\left(^{\circ}\right)$ & $\begin{array}{c}L(\AA) \\
(+/-50 \AA)\end{array}$ \\
\hline Expérience 1 : & & & & \\
verre & 16,33 & 0,32 & 0,29 & 280 \\
silicium (100) & 16,32 & 0,33 & 0,30 & 270 \\
Expérience 2: & & & & \\
alumine $\alpha$ & 16,52 & 0,40 & 0,38 & 220 \\
silicium (100) & 16,53 & 0,34 & 0,31 & 260 \\
\hline
\end{tabular}

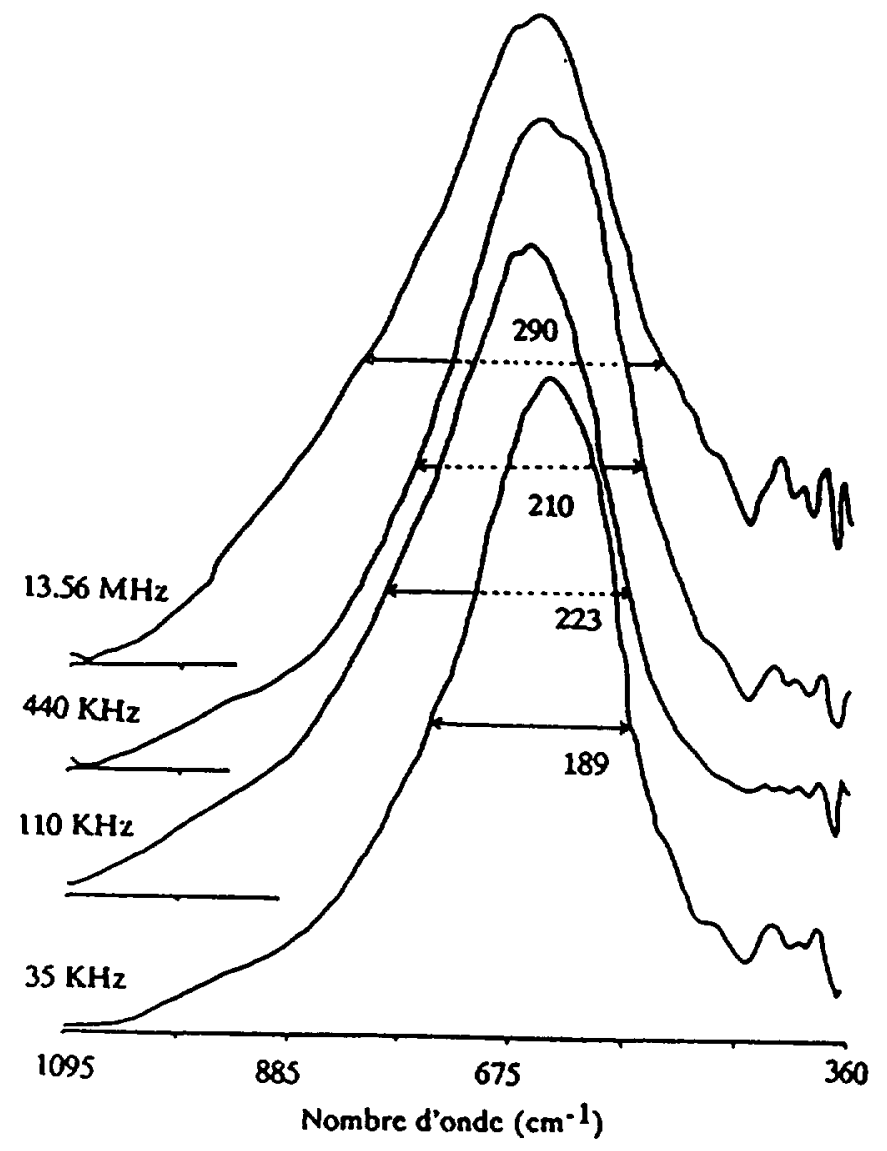

Fig. 7. - Elargissement de la bande de vibration de l'oscillateur Al-N.

[Broadening of Al-N vibrating bond.] 


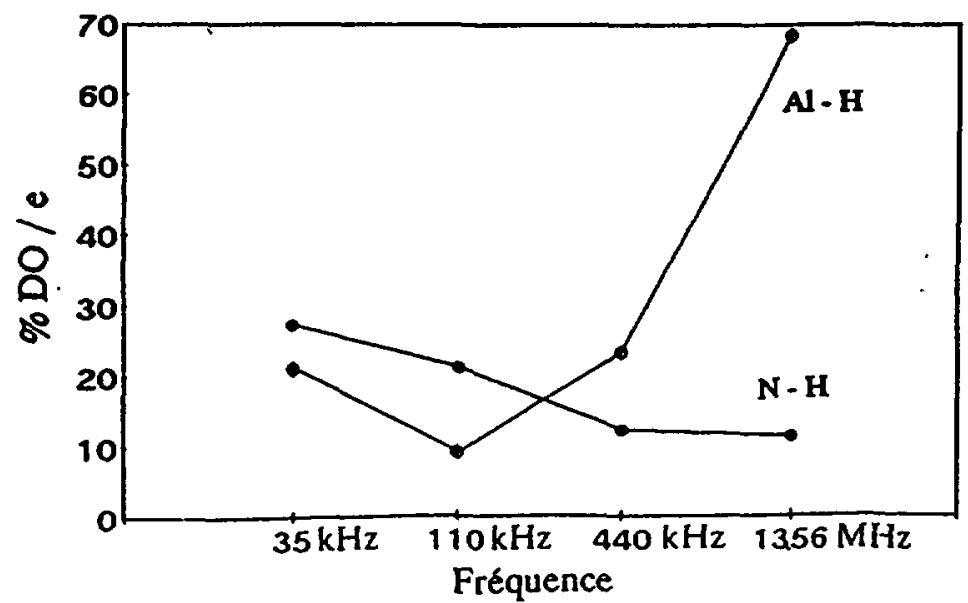

Fig. 8. - Variation de l'intensité des bandes d'absorption des vibrateurs Al-H et N-H.

[Intensities of Al-H and N-H bonds observed in IR spectroscopy.]

dans ce matériau de structure amorphe dans ces conditions. Cependant, il convient de noter que la quantité totale d'hydrogène dans les différents films est identique quelle que soit la fréquence d'excitation utilisée pour leur synthèse.

4.4 SPECTROSCOPIE DES ÉLECTRONS AUGER. - Nous nous sommes intéressés à l'évaluation de la concentration moléculaire dans les films de nitrure d'aluminium. Ces concentrations ont été obtenues à partir des enregistrements des spectres Auger $E . N(E)$ dans la zone $50-520 \mathrm{eV}$ pour les transitions $N$ KLL à $375 \mathrm{eV}$ et $1350-1450 \mathrm{eV}$ pour les transitions $\mathrm{AlKL}_{23} \mathrm{~L}_{23}$ à $1390 \mathrm{eV}$. Le nitrure de silicium et l'aluminium sont pris comme références. L'intensité des deux pics est déterminée à partir de la mesure de leur hauteur par rapport au fond continu. La première observation relative aux résultats du tableau III montre que les écarts entre les valeurs

Tableau III. - Concentrations molaires dans les films $\mathrm{Al}_{\checkmark} \mathrm{N}_{v}$ préparés à différentes fréquences.

[Molecular concentrations in $\mathrm{Al}_{1} \mathrm{~N}$, films.]

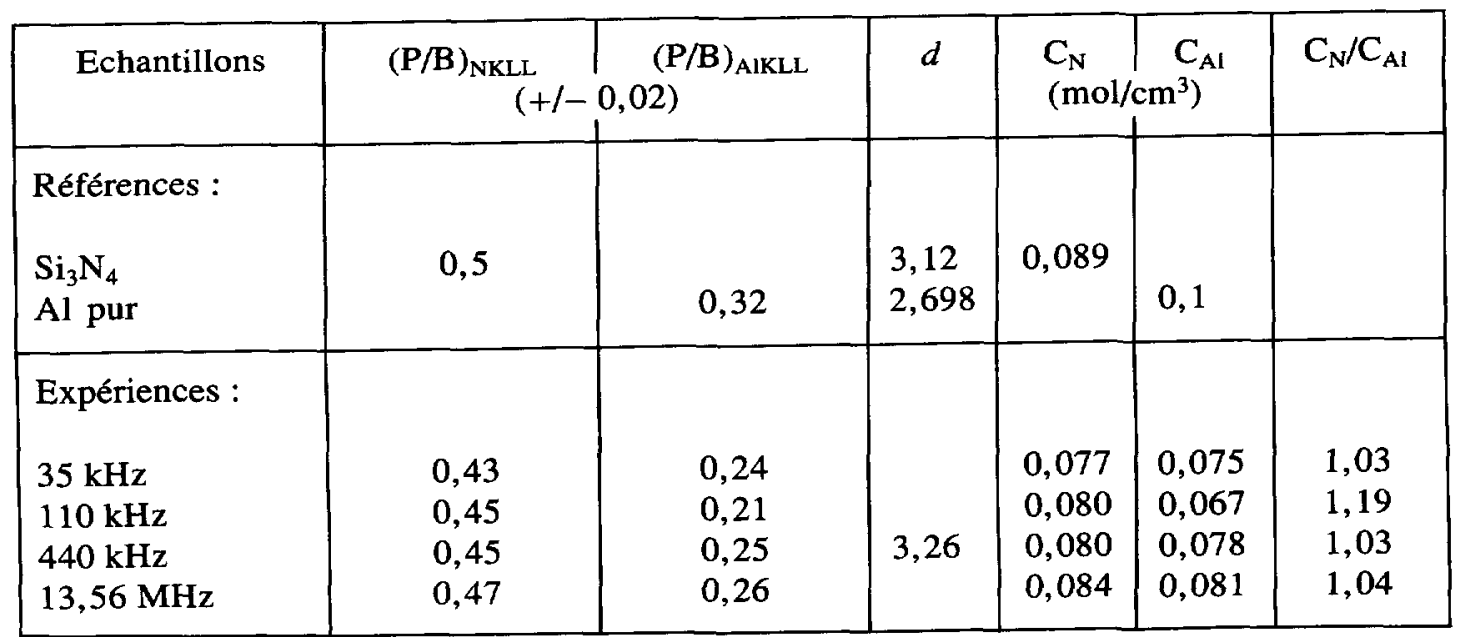


des P/B sont très faibles, aussi bien pour l'azote que pour l'aluminium. Ceci indique que les nitrures synthétisés ont des compositions très voisines quelle que soit la fréquence d'excitation utilisée. Seul le film obtenu à $110 \mathrm{kHz}$ présente un excès d'azote (ou un défaut d'aluminium) qui rend compte du déplacement du pic de diffraction vers les plus grandes valeurs de $d$.

\section{Interface AIN/Si.}

Dans cette partie nous nous intéressons à la caractérisation de l'interface AlN/Si par microscopie en transmission et à la détermination de sa composition chimique par spectroscopie des électrons Auger.

L'observation d'un échantillon préparé à $440 \mathrm{kHz}$ (Fig. 9) montre des cristallites de taille régulière $(1000 \times 200 \AA)$ dont 1'axe principal de croissance est perpendiculaire à la surface du substrat. Une ligne claire de largeur homogène (100 $\AA$ ) reproduit parfaitement la surface du silicium et marque l'interphase. Une approche en haute résolution nous a permis d'obtenir de plus amples informations sur cette interphase. L'apparence mouchetée de cette zone est caractéristique d'un matériau amorphe dont l'épaisseur est de $90 \AA$. Par contre, dans la couche de nitrure d'aluminium, la présence de franges d'interférences d'égale épaisseur traduit le caractère cristallin du dépôt. En plus on peut mettre en évidence une région intermédiaire d'environ $200 \AA$, proche de l'interphase, où les cristallites sont de petite taille ( $80 \AA$ ) et sans orientation préférentielle. La croissance cristalline se fait donc à partir de cette interphase amorphe, dont la présence explique la non influence du substrat sur l'orientation préférentielle

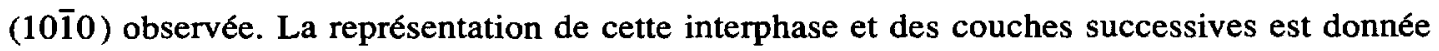
sur la figure 10.

La zone d'environ 150-200 $\AA$ comprenant les couches amorphe et microcristalline a été analysée par spectroscopie Auger. L'interphase est révélée en $15 \mathrm{~min}$ par abrasion ionique $\left(\mathrm{Ar}^{+}\right.$de $\left.3 \mathrm{keV}\right)$. Elle est constituée des seuls éléments $\mathrm{Al}, \mathrm{N}$, $\mathrm{O}$ et $\mathrm{Si}$ dont la répartition en profondeur est représentée sur la figure 11 dans laquelle la notation «épaisseur 0 » indique le début de l'interphase amorphe par l'apparition de l'élément oxygène après $100 \mathrm{~min}$ de

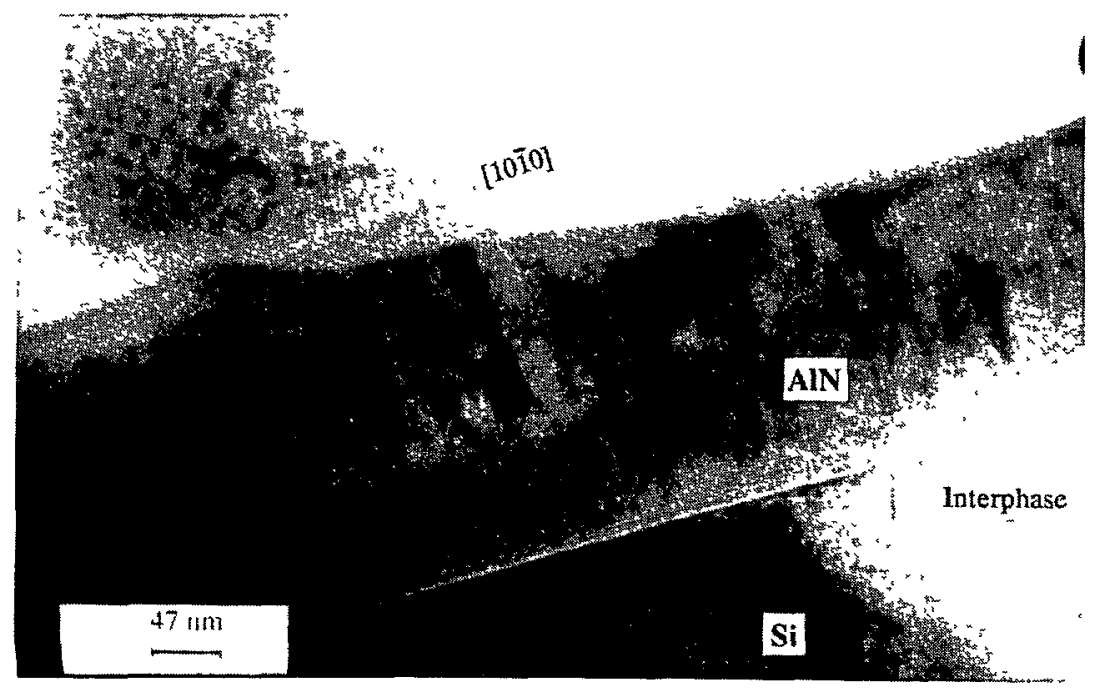

Fig. 9. - Image en champ clair des cristallites d'AlN synthétisé à $440 \mathrm{kHz}$.

[Bright field image showing AlN crystallites synthetized at $440 \mathrm{kHz}$.] 


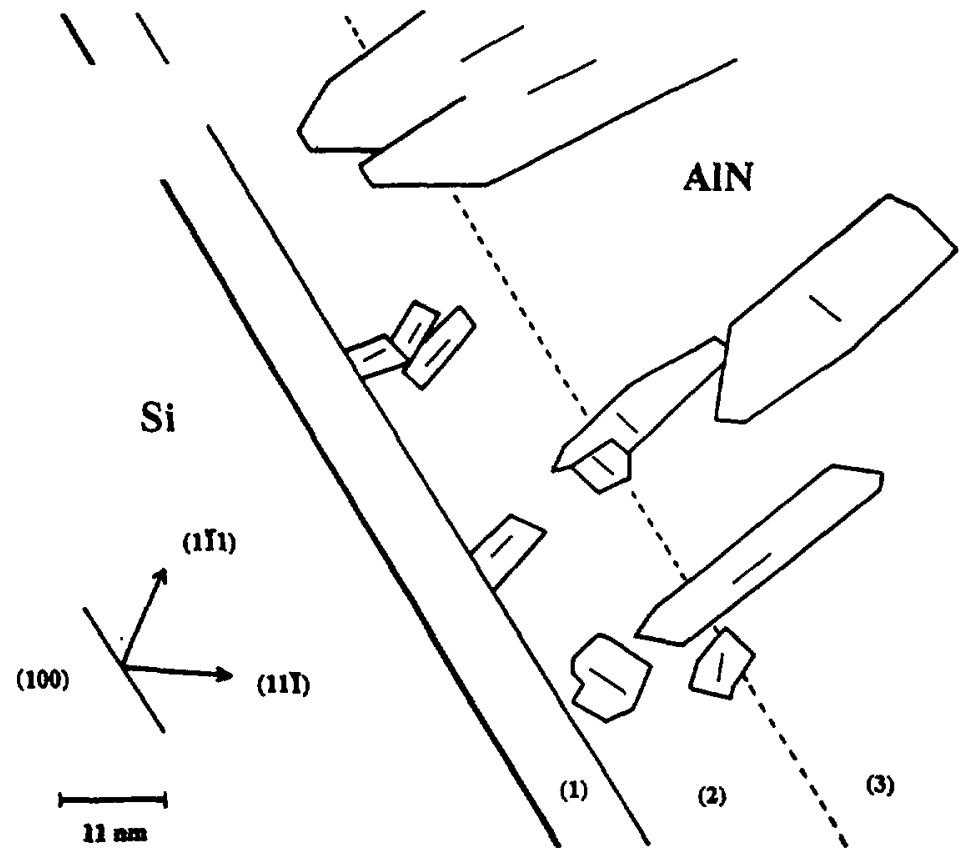

Fig. 10. - Représentation schématique des différentes zones de l'interphase AlN/Si. (1) Interphase amorphe Si/AlN. (2) Zone intermédiaire de petites cristallites non orientées. (3) Cristallites orientées perpendiculairement au substrat.

[Diagram of organization of AlN crystallite growth. (1) Si/AlN amorphous interface. (2) Intermediate zone of small crystallites. (3) Oriented crystallites.]

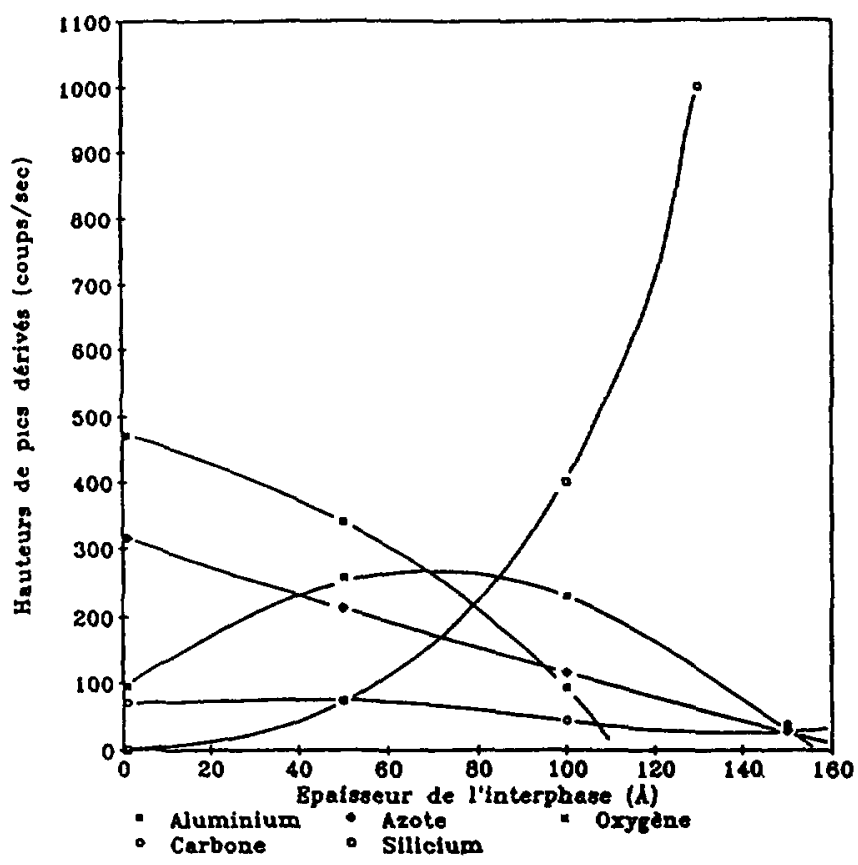

Fig. 11. - Profils de concentration des différents éléments de l'interphase AIN/Si.

[Element depth profiles in the AIN/Si interphase region.] 
décapage. Les spectres $E . N(E)$ sont représentés sur la figure 12 . Le spectre (après $30 \mathrm{~min}$ ) est celui du nitrure d'aluminium, tandis que le dernier (après $120 \mathrm{~min}$ ) est celui du silicium. Plusieurs aspects apparaissent sur les trois spectres relatifs à l'interphase. L'élargissement de la raie $\mathrm{Al} \mathrm{LVV}$, simultanément à l'augmentation de la quantité d'oxygène, dénote la présence de liaison Al-O. De plus, sur le spectre observé après 105 min de décapage, la présence d'un pic à $78 \mathrm{EV}$ (correspondant à la liaison $\mathrm{Si}-\mathrm{O}$ ) de très faible intensité, indique que l'oxygène est préférentiellement lié à l'aluminium. Par contre, après $110 \mathrm{~min}$ d'érosion ionique, le silicium se retrouve principalement sous la forme silicium pur ( $\mathrm{Si}-\mathrm{Si}$ à $93 \mathrm{eV}$ ).

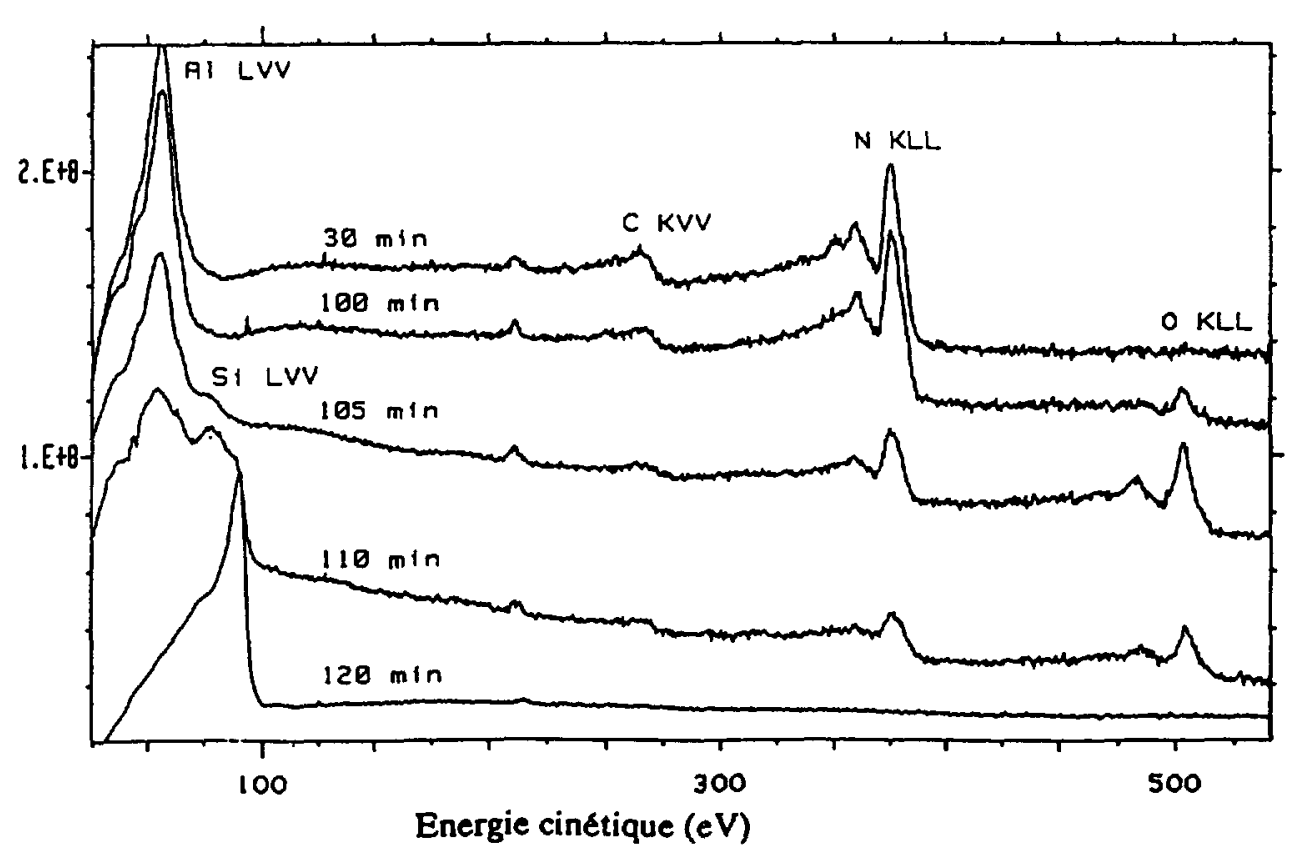

Fig. 12. - Spectres basses énergies $E .(N E)$ obtenus au cours de l'érosion ionique de l'interphase AlN/Si.

[E. (NE) low energy spectra after sputtering of the interphase AIN/Si.]

L'observation des spectres hautes énergies (Fig. 13) confirme la présence de silicium pur, tandis que la raie Al KLL peut aussi bien correspondre aux liaisons Al-N (1 388,9 eV dans AlN) qu'Al-O (1 $388 \mathrm{eV}$ dans $\mathrm{Al}_{2} \mathrm{O}_{3}$ ).

On observe donc l'oxydation de l'aluminium par l'oxygène initialement présent à la surface du silicium (couche d'oxyde natif de 20 à $30 \AA ̊$ ). Ceci peut être expliqué par deux phénomènes :

- réduction de l'oxyde de silicium par l'aluminium, cohérente avec la valeur de l'enthalpie libre de formation de $\mathrm{Al}_{2} \mathrm{O}_{3}$ plus faible que celle de $\mathrm{SiO}_{2}$;

- diffusion de l'oxygène à partir de la surface du substrat vers le nitrure d'aluminium au début de la croissance.

L'interphase film-substrat est donc constituée de tous les éléments présents dans la couche, le substrat et sa surface, soit $\mathrm{Al}, \mathrm{Si}, \mathrm{N}$ et $\mathrm{O}$, avec un gradient de composition. 

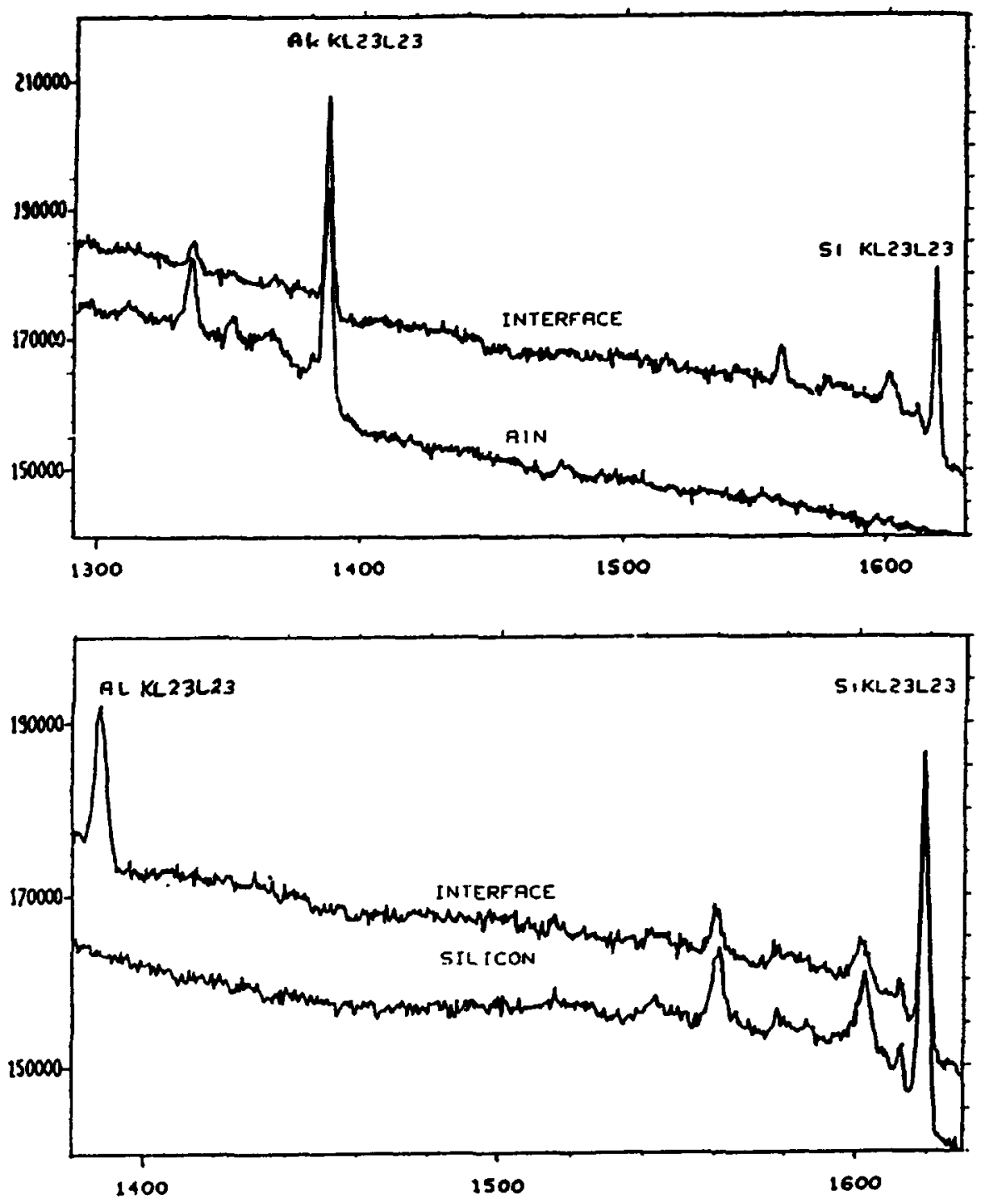

Fig. 13. - Comparaison des spectres hautes énergies de l'interphase avec AlN et Si.

[High energy spectra of the interphase.]

\section{Conclusions.}

La méthode de dépôt chimique en phase vapeur assisté d'un plasma réactif permet la réalisation de couches de nitrure d'aluminium de très bonne pureté, homogènes en composition et en épaisseur. Ces couches présentent également de très bonnes qualités de recouvrement et d'adhérence. Selon les conditions expérimentales de dépôt et, principalement, selon la fréquence d'excitation plasma, la dimension des cristallites peut varier dans une large plage et la direction préférentielle de croissance de ces cristallites peut être modifiée par l'utilisation de températures de croissance différentes, comme le montre le tableau IV. 
Tableau IV. - Evolution de la cristallinité des films de nitrure d'aluminium en fonction de la température de croissance et de la fréquence d'excitation plasma.

[Influence of growing temperatures and frequency on AlN coating crystallinity (CVD and PECVD results).]

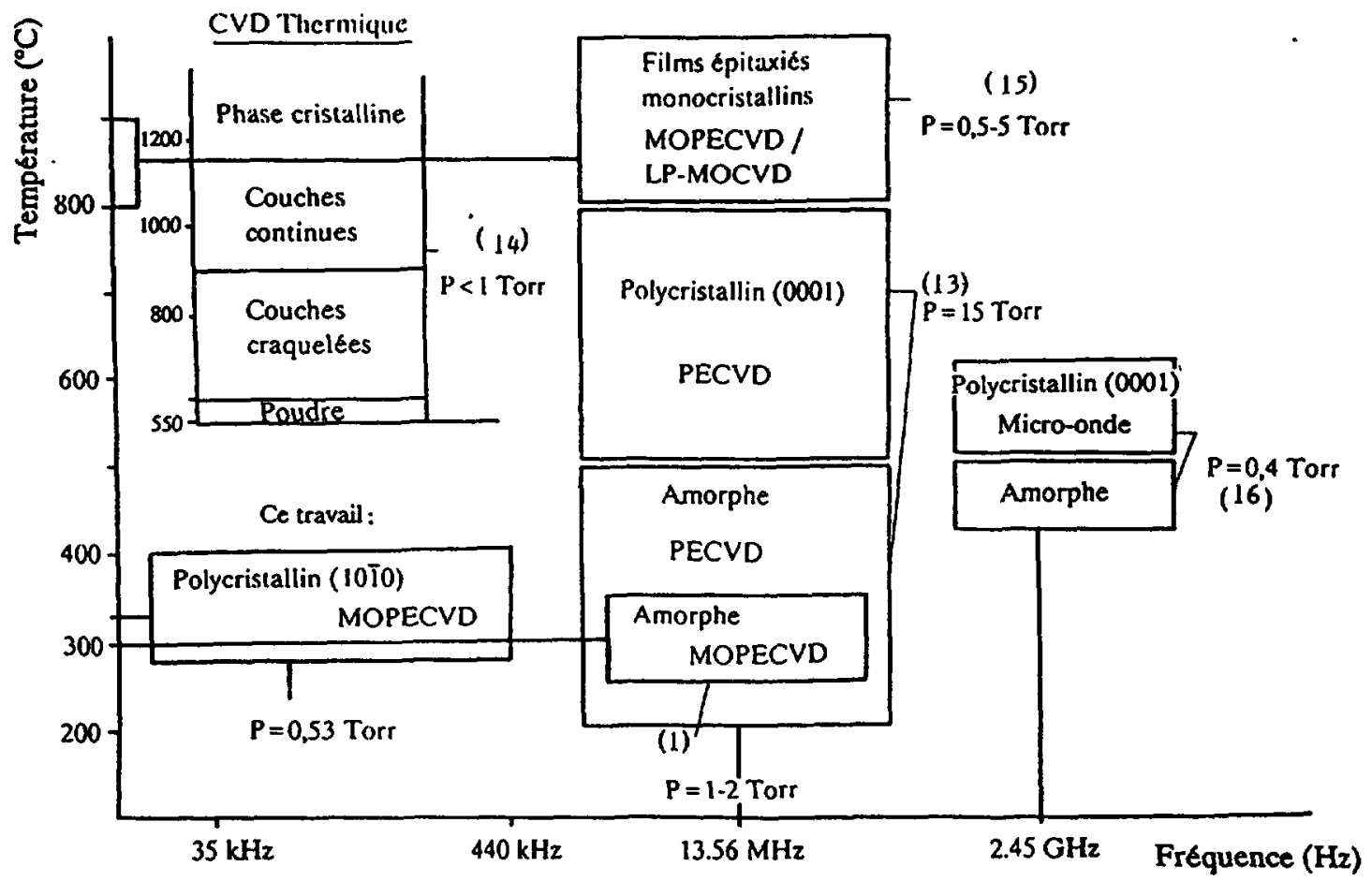

Une interphase amorphe de faible épaisseur a été mise en évidence par microscopie en transmission et spectroscopie des électrons Auger.

Les caractéristiques des couches et de leur interface avec un substrat font du nitrure d'aluminium un matériau d'avenir pour de multiples applications.

\section{Bibliographie}

[1] Hasegawa F., Takahashi T., Kubo K., Nannichi Y., Jpn J. Appl. Phys. 26 (9) (1987) 1555.

[2] WRIEDT H. A., Binary Alloy Phase Diagrams 1, 135.

[3] Cox G. A., Cummins D. O., Kawabe K., Tredgold R. H., J. Phys. Chem. Solids 28 (1967) 543.

[4] Yim W. M., Stofko E. J., Zanzucchi P. J., EtTenberg M., Gilbert S. L., J. Appl. Phys. 44 (1973) 292.

[5] Graupner P., Pommier J. C., Cachard A., Coutaz J. L., J. Appl. Phys. 71 (9) (1992) 4136.

[6] Dobrynin A. V., Naida G. A., Novoselov V. A., Phys. Stat. Sol. (a) 104 (1987) K47.

[7] Fuji Y., Yoshida S., Misawa S., Maekawa S., Sakudo T., Appl. Phys. Lett. 31 (12) (1977) 815.

[8] FEIL M., Hybrid Circuits 18 (1989) 29.

[9] Kurokawa Y., Utsumi K., Takamizawa H., Kamata T., Noguchi S., IEEE Trans. on components, hybrids and manufacturing technology, CHMT 8 (2) (1985) 247.

[10] Azéma N., Durand J., Berjoan R., Dupuy C., Cot L., J. Eur. Ceram. Soc. 8 (5) 291.

[11] Anthony R. W., Solid State Chemistry and its Applications (J. Wiley and Sons, 1984) p. 174. 
[12] Matsuda A., Kumagi K., Tanaka K., Symp. on ISIAT' 83, Kyoto, Japan (1983).

[13] IтоH H., Kato M., SugiYamza K., Thin Solid Films 146 (1987) 255.

[14] SuzuKi M., TanjI H., Proceedings 10th Internat. Conf. on CVD (1987) 1089.

[15] SaTo K., Ultrasonics Symposium (1985) 192.

[16] Someno Y., Sasaki M., Hirai T., Jpn J. Appl. Phys. 29 (2) (1990) L358. 